\begin{tabular}{|c|c|c|}
\hline 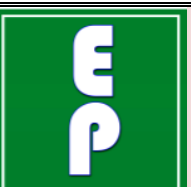 & $\begin{array}{l}\text { International Journal of Current Research } \\
\text { and Academic Review }\end{array}$ & 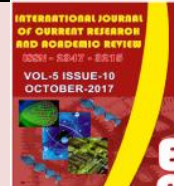 \\
\hline $\begin{array}{l}\text { EXCELLENT } \\
\text { PUBLISHERS } \\
\end{array}$ & Journal homepage: http://www.ijcrar.com & \\
\hline
\end{tabular}

doi: https://doi.org/10.20546/ijcrar.2017.510.002

\title{
Neurocysticercosis: Trends in Diagnosis and Management
}

\author{
Ramandeep Kaur ${ }^{1}$, Harit Kumar ${ }^{2 *}$, Shalini Pathania ${ }^{1}$, and Nitin Goel Insan ${ }^{2}$ \\ ${ }^{I} M M$ Institute of Nursing-MMU, Mullana, Ambala, Haryana, India \\ ${ }^{2}$ Department of Microbiology, MMIMSR-MMU, Mullana, Ambala, Haryana, India
}

*Corresponding author

\begin{abstract}
Neurocysticercosis (NCC) is a major cause of neurological morbidity in the world. The diagnosis of NCC is challenging in endemic and resource-limited countries where laboratory and imaging techniques are not available. Clinical manifestations are nonspecific, most neuroimaging findings are non-pathognomonic, and some serologic tests have low sensitivity or specificity. The treatment of the NCC includes cysticidal drugs (e.g., albendazole and praziquantel), and neurosurgical procedure, depending upon the situation. Currently, there are several diagnostic and management issues which remain unresolved. This review will help to look the recently going diagnostic procedures and the medical management of the disease.
\end{abstract}

\section{Article Info}

Accepted: 04 September 2017

Available Online: 20 October 2017

Keywords

Neurocysticercosis,

Taeniasolium,

Diagnosis and Management.

\section{Introduction}

Neurocysticercosis (NCC), a parasitic infection of the nervous system caused by the encysted larval stage of the tapeworm Taenia solium. Neurocysticercosis is considered by the World Health Organization to be the most common preventable cause of epilepsy in the developing world, with an estimated 2 million people having epilepsy caused by $T$. solium infection. ${ }^{(1,2,3)}$

Human is the only definitive host of $T$. solium harboring adult tapeworm in the intestine (taeniasis), whereas both man and pig can act as intermediate hosts and harbor the larvae in different internal organs (cysticercosis) including brain (NCC). ${ }^{(4,5)}$

\section{Epidemology}

NCC was first reported in a coolie from Madras, who died due to seizure and was found to be infected with cyst on autopsy. ${ }^{(6)}$ In 1934, high rate of new onset epilepsy related to cysticercosis in the British army deployed in India was noticed. ${ }^{(7)}$ Estimation of the prevalence of NCC in the general population is challenging because it is difficult to perform neuroimaging in a large population. Moreover, poor record keeping in several developing countries and socioeconomic issues gets adds to this also. ${ }^{(8)} \mathrm{NCC}$ is asymptomatic in approximately $50 \%$ of cases. ${ }^{(9)}$

\section{Disease burden in India}

All the biological markers for transmission of $T$. solium taeniasis and cysticercosis exist in India. There are great disparities within the country in geography, ethnicity religion rituals, income, food habits, personal hygiene, level of education and standards of living, which are likely to influence the disease burden. ${ }^{(10)}$ Consequently there are wide variations in the frequency of cysticercosis in India shown in Figure 1. 
In a community survey of 50,617 individuals from South India, the prevalence of active epilepsy was 3.83 per 1000 and NCC was detected in $28.4 \%$ of them by CT. ${ }^{(11)}$

In a study based on 30 cluster sampling approach suggested by WHO in the rural pig farming community of Mohanlalganj block, Lucknow district, Uttar Pradesh, the prevalence of taeniasis was $18.6 \%$; factors associated with taeniasis were age above 15 years.

In the same community active epilepsy was identified and clinically confirmed in $5.8 \%$ of the populations during door to door survey and $48.3 \%$ of them fulfilled either definitive or probable diagnostic criteria of NCC. ${ }^{(12)}$

\section{Life cycle}

Taenia solium completes its life cycle in two hosts-

Man: Definitive host

Pig: Intermediate host

\section{Clinical picture}

It depends on the site involved. Any organ or tissue may be affected but the most common being subcutaneous tissues, muscles, Central nervous system (CNS) and eyes.

Neurocysticercosis is the most common cause of epilepsy in the world. It is of two types-

Parenchymal: Involves brain parenchyma

Extraparenchymal: Involves meninges, ventricles and spinal cord

Manifestations are mainly due to the calcified form of cysticercus cellulosae leading to behavioural disorders, increased intracranial pressure, chronic meningitis, hydrocephalus.

\section{Laboratory diagnosis}

\section{Biopsy:}

Histological confirmation of neurocystecercosis in most of the cases is not possible. However, Detection of the parasite in a biopsy specimen of skin nodules may also aid in the diagnosis. ${ }^{(14,15)}$

\section{Imaging methods:}

The mainstay of diagnosis of NCC is neuroimaging using contrast enhanced computerized tomography (CECT) or magnetic resonance imaging (MRI). CT scan is preferred for identifying parenchymal calcifications while MRI is the preferred modality for parenchymal lesions which are in the temporal lobe and frontal lobe close to the skull base, intraventricular cysts and subarachnoid cysts. ${ }^{(16)}$

While CT and MRI will almost invariably identify the NCC lesions, there may be diagnostic difficulties in differentiating NCC from other lesions of the brain such as tuberculomas, other parasitic cysts such as hydatid cysts, neoplastic lesions such as gliomas and metastatic lesions. ${ }^{(17)}$

\section{Serological tests:}

A major disadvantage of this approach is that false positive results can be obtained as antibodies do not necessarily indicate an active infection whereas presence of antigens indicates an active infection. ${ }^{(18,19,20)}$

\section{a. Enzyme Linked Immunotransfer Blot (EITB)}

This assay tests for antibodies to seven larval specific antigens. It has a sensitivity of $98 \%$ and specificity of $100 \%$ in patients with more than one live cyst or subarachnoid disease. ${ }^{(21)}$ This technique uses lentil lectin purified glycoprotein antigens to detect antibodies to $T$. solium in serum. ${ }^{(22)}$

\section{b. Enzyme Linked Immunosorbent Assay (ELISA)}

Antibody detection: It can detect IgG antibody against parasite antigens in serum or CSF sample, although IgA, $\mathrm{IgE}$, and $\operatorname{IgM}$ can also be detected but have little value in diagnosis. ${ }^{(23,24)}$

In a study, Anti-T. solium Ab levels were determined by an in-house ELISA.

Vesicular fluid was recovered from $T$. solium cysticerci and used as the source of Antigen. ${ }^{(25)}$

Dot ELISA: The Dot- ELISA constitutes a nitrocellulose membrane bound with the cysticercus antigen, the specific cysticercus antibodies, if present in the serum, will bind to the antigen and is detected visually by addition of an enzyme-labeled second antibody. ${ }^{(26)}$ 
Antigen Detection: Enzyme Linked Immunosorbent Assay detects circulating antigens from the $T$. solium metacestode (B158/B60 Ag-ELISA or HP10 Ag-ELISA). ${ }^{(27)}$

It is almost always positive in patients with subarachnoid disease but like the EITB, is likely to be negative in those with calcific lesions and with one or two parenchymal lesions. ${ }^{(1)}$

The Ag-ELISA could be used to monitor the effects of therapy as its levels fall rapidly following successful therapy. ${ }^{(28)}$

\section{Molecular methods}

Polymerase Chain Reaction (PCR): This technique detects the DNA of $T$. solium in the sample. The target element is pTsol9, which is highly repetitive in parasite genome. A study was done by Michelet et al., (2011) they used primers 5-CAGGGTGTGACGTCATGG-3 (forward primer; positions 21 to 38, 179 to 196, or 336 to 353) and 5-GCTAGGCAACTGGCCTCCT-3 (reverse primer; positions 122 to 140,280 to 298 , or 437to 455) for the amplification of pTsol9 and found PCR $80 \%$ specific for the diagnosis of NCC. ${ }^{(29)}$

\section{Management}

The initial approach to the management of the cysticercosis includes symptomatic treatment as well as depends upon the viability of the parasite. As the agent of the cysticercosis affects four major systems of the human body (Skin, Muscles, Nervous system \& Ocular system).

\section{Medical management}

The treatment of choice for medications includes: ${ }^{(30)}$

(a) Larvicidal agents

(b) Corticosteroids

(c) Antiepileptic Drugs

\section{(a) Larvicidal agents}

This drug is given only if the larval agent is viable. This is administered to kill the larval agent. It includes:

Praziquantel is a quinolone which produces spastic paralysis of the parasite musculature and destroy the Scolex. ${ }^{(31)}$
Albendazole is an imidazole which acts by inability the uptake of glucose by parasitic membranes thus causing energy depletion. ${ }^{(32)}$

\section{(b) Corticosteroid medication}

It is used to treat inflammation. Prednisolone and dexamethasone are the drugs of choice.

\section{(c) Antiepileptic drugs}

It is helpful to control the seizures.

Example includes Phenytoin and Carbamazepine.

\section{Surgical management}

The surgical management is according to the affected area of body as follows: ${ }^{(33)}$

(a) Cyst in skin/muscle: If the cyst is present under the skin or muscle then surgical excision is necessary to treat this condition.

(b) Cyst in eye: If the cyst is located in anterior chamber than Viscoexpression and for posterior chamber, Vitrectomy is the surgery of choice.

(c) Cyst in nervous system (Brain and spinal cord): Ventriculo-peritoneal shunt placement is needed, if the cyst develops into the ventricles of the brain causing increased intracranial pressure.

\section{Nursing management}

Nurses play a key role in the care of patients with neurocysticercosis.

The first step of the nursing management is assessment which includes history taking and performing an accurate physical/neurological examination to recognize changes.

\section{Nursing diagnosis}

\section{Preoperative nursing diagnosis}

Headache related to increased intracranial pressure secondary to hydrocephalies as evidenced by pain scale.

Risk of injury related to seizures secondary to disease condition as evidenced by verbalization with the patient.

Visual impairment related to ocular cyst. 
Fig.1 Geographical distribution of cysticercosis and Taeniasoliumtaenaisis in India ${ }^{(10)}$

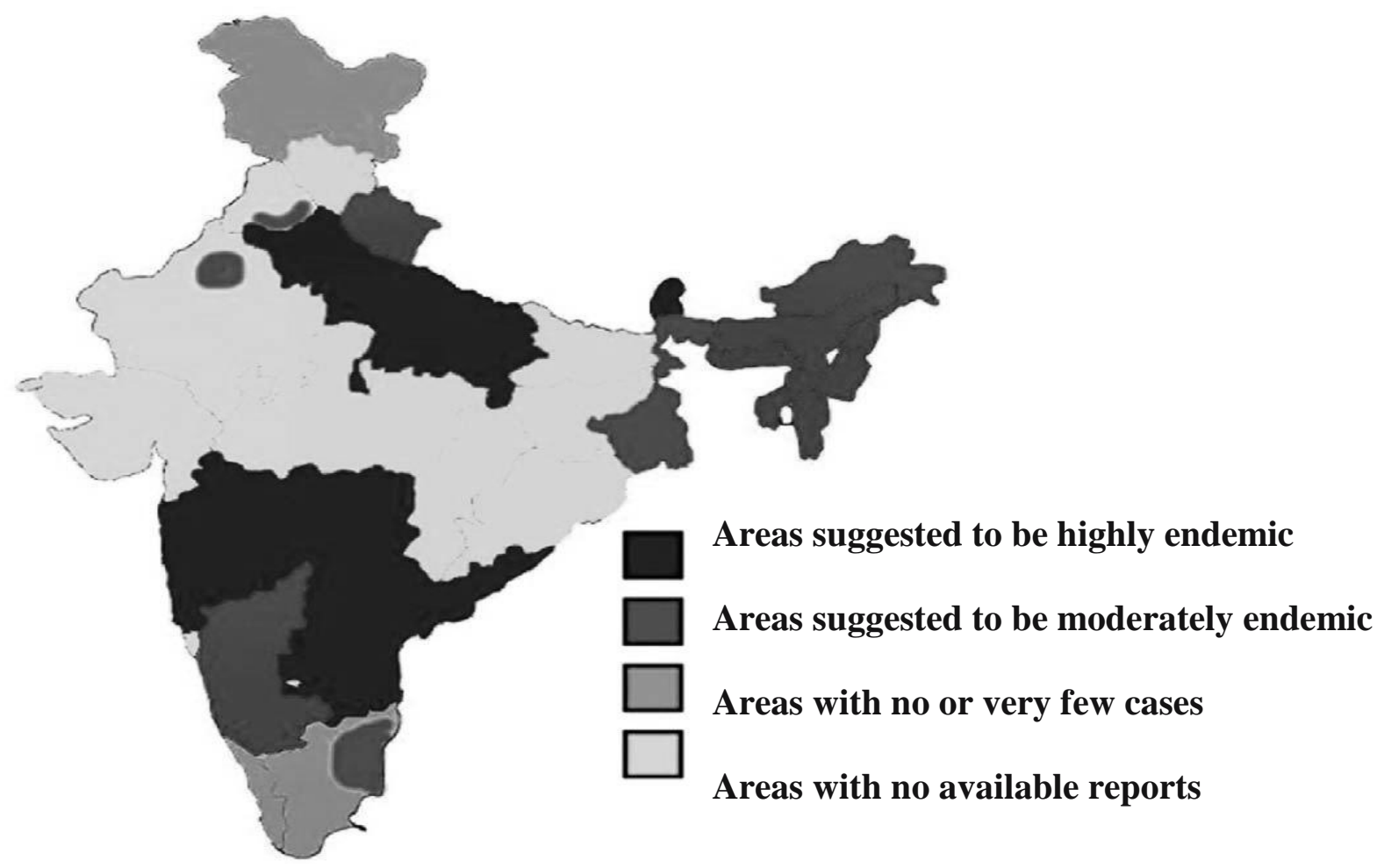

Fig.2 Life cycle of $T$. solium ${ }^{(13)}$

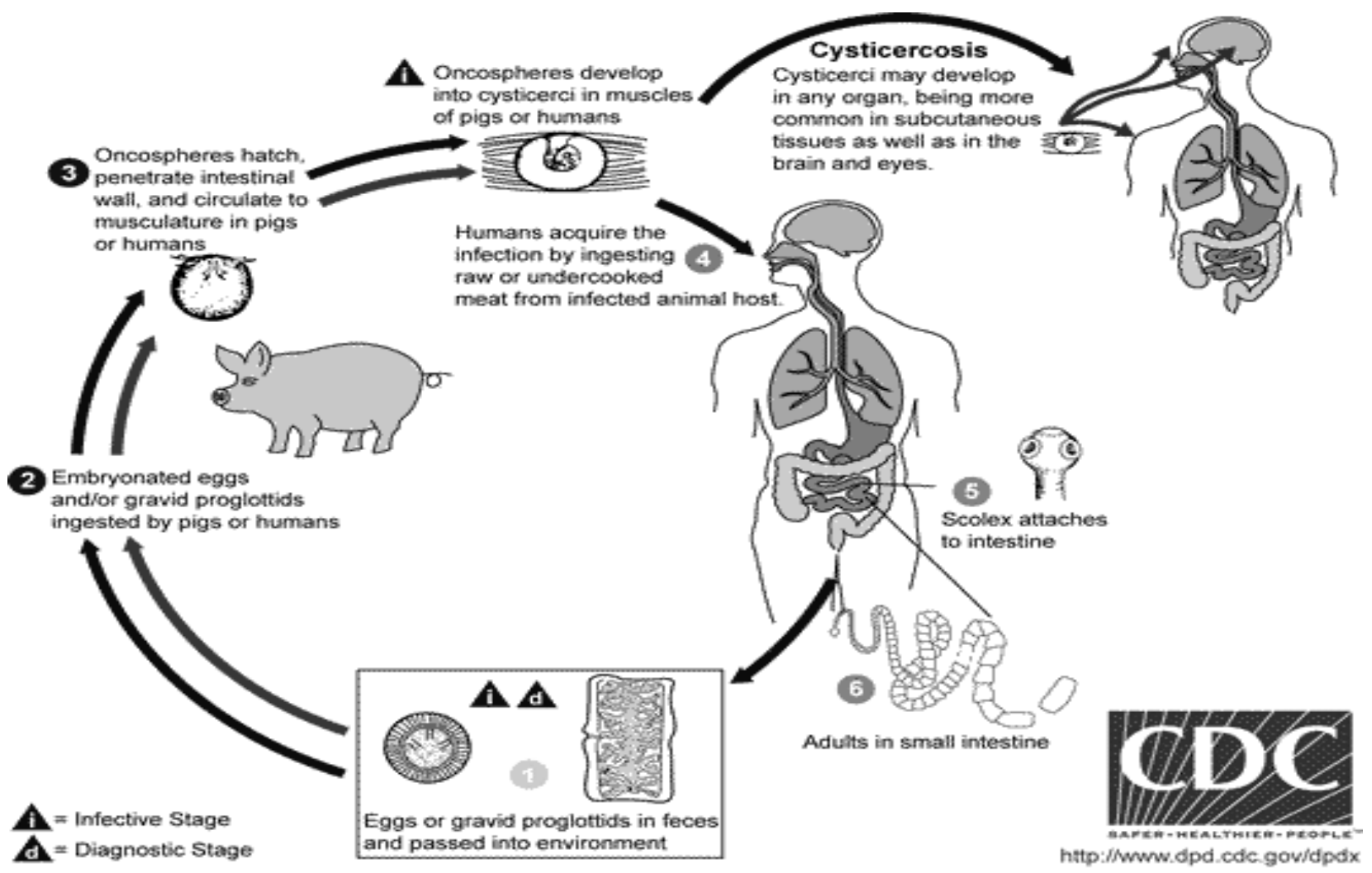


Fig.3 A driven equilibrium sequence axial magnetic resonance image showing a fourth ventricular cysticercal cyst with the scolexseen as a hypointense (dark) dot (arrow)

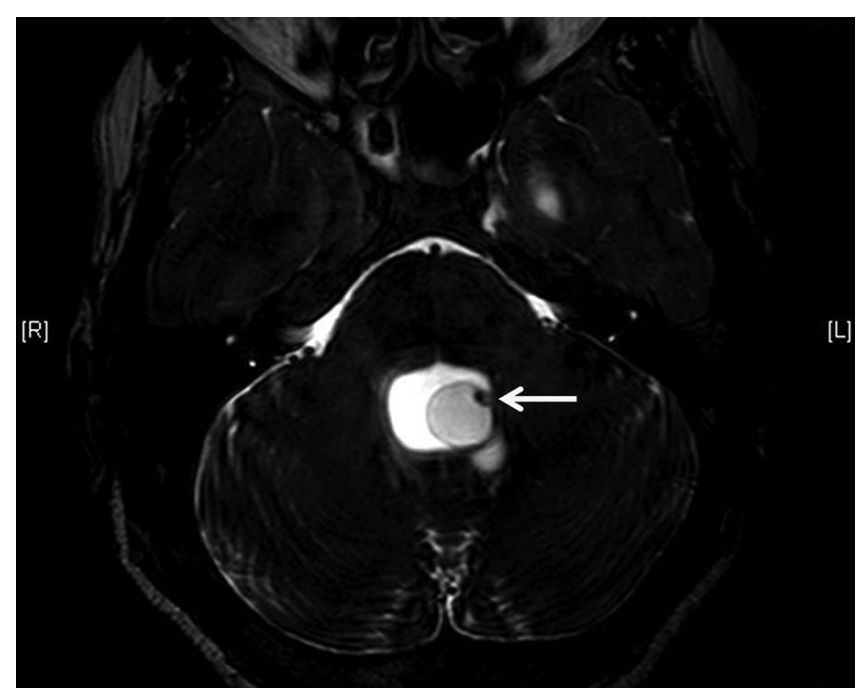

Fig.4 1.Unenhanced CT showing multiple active vesicular cysts. 2. contrast-enhanced CT showing a similar ringenhancing lesion probably corresponding to the granular-nodular pathologic stage. Epilepsia (C ILAE
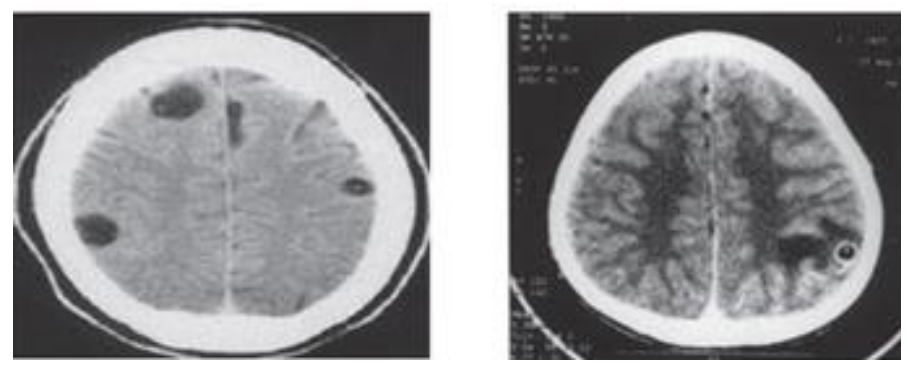

Medical Management

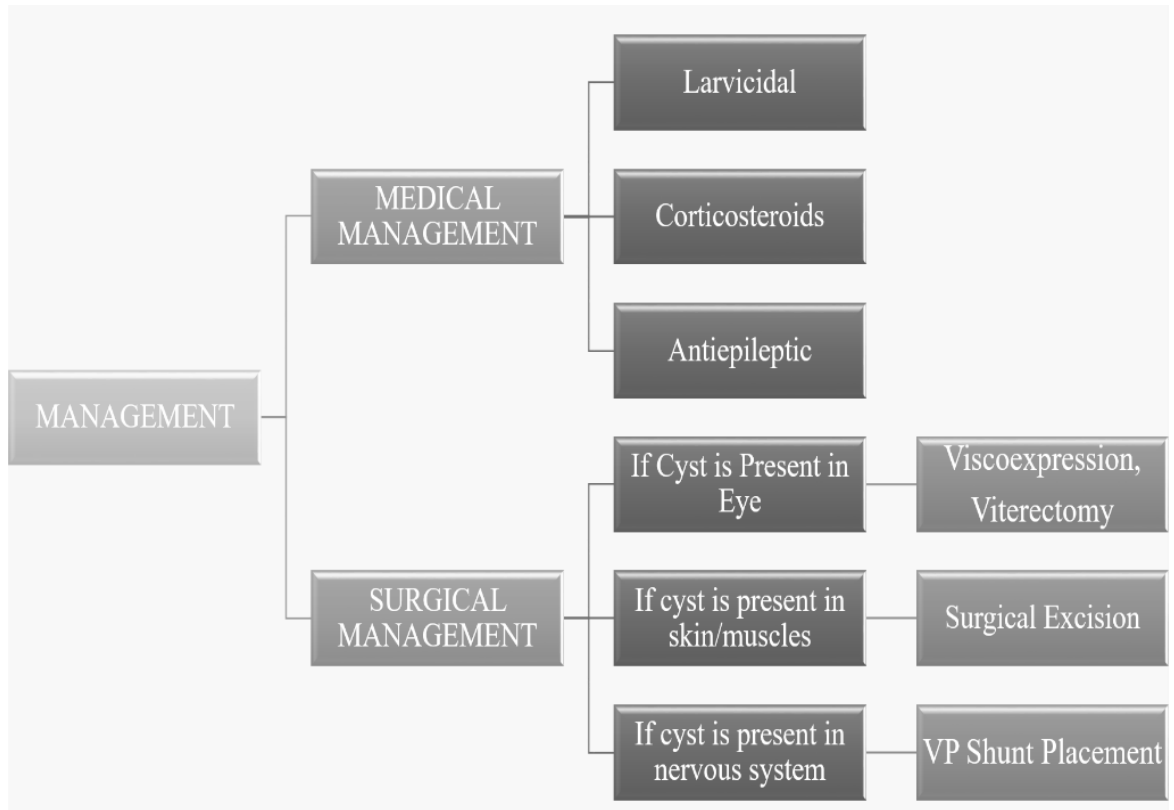




\section{Postoperative nursing diagnosis}

Pain related to surgical procedure as evidenced by facial expression of the patient.

Risk of infection related to surgical procedure as evidenced by vital signs of the patient.

Knowledge deficit related to treatment and prognosis of disease.

\section{Prevention}

Wash hands with soap and warm water after using the toilet, changing diapers, and before handling food

Wash and peel all raw vegetables and fruits before eating

Use fresh food and water safety practices while travelling in developing countries such as:

Drink only bottled or boiled (1 minute) water

Avoid eating meat: Since pork is the prime culprit for spreading $T$. solium, their eggs can be found on meat that was stored or transported alongside pork.

Cook the meat properly to destroy potential infestation of $T$. solium.

Proper disposal of feces and prevention of contamination with food given to pigs is also important.

It is concluded that NCC is the most common parasitic infection of the CNS and identified as the most important cause of acquired active epilepsy.

However there is a lack of highly sensitive, specific and cost-effective tools for diagnosis of NCC but there is also progress in development of immunological tests in the last two decades. In the diagnosis of NCC neuroimaging still remains the central. Appropriate measures like health education, mass awareness, better medical facilities, mass treatment of $T$. solium carriers, and restriction on sale of measly pork may help in reducing the disease burden in the endemic areas.

The management of NCC is debated especially with respect to the use of cysticidal drugs as their benefits have not been conclusively proven. There are several other management issues awaiting resolution.

\section{References}

1. Garcia HH, Del Brutto OH. Neurocysticercosis: updated concepts about an old disease. Lancet Neurol. 2005; 4:653-61.

2. Singh G, Burneo JG, Sander JW. From seizures to epilepsy and its substrates: neurocysticercosis. Epilepsia. 2013; 54:783-792.

3. Wallin MT, Kurtzke JF. Neurocysticercosis in the United States. Review of an important emerging infection. Neurology. 2004; 63:1559-64.

4. Del Brutto OH. Neurocysticercosis among international travellers to disease-endemic areas. J Travel Med. 2012; 19:112-7.

5. Garcia-Noval J, Allan J C, Fletes C, Moreno E, DeMata F, Torres- Alvarez R, Soto de Alfaro H, Yurrita P, Higueros-Morales H, Mencos F and Craig P S. Epidemiology of Taenia solium taeniasis and cysticercosis in two rural Guatemalan communities; Am. J. Trop. Med. Hyg. 1996: 55:282-289

6. Armstrong H. A case of Cysticercuscellulosaeof brain in a native coolly; Indian Med. Gaz1888: 23:252.

7. MacArthur W P. Cysticercosis as a cause of epilepsy in man; Trans. R. Soc. Trop. Med. Hyg. 1934: 26:525-528

8. Fleury A, Gomez T, Alvarez I, et al., High prevalence of calcified silent neurocysticercosis in a rural village of Mexico. Neuroepidemiology. 2003; 22:139-145.

9. Winkler AS, Richter H. Landscape analysis: management of neurocysticercosis with an emphasis on low- and middle-income countries (LMIC). Available from: http://apps.who.int/iris/bitstream/ 10665/152896/1/WHO_HTM_NTD_NZD_2015.05 _eng.pdf.( Accessed on $24^{\text {th }}$ Feb, 2017).

10. Prasad KN, Prasad A, Verma A, Singh AK. Human cysticercosis and Indian scenario: a review. J. Biosci. 2008: 33(4):571-582.

11. Rajshekhar V M, Raghava V, Prabhakaran V, Ommen A and Muliyil J. Active epilepsy as an index of burden of neurocysticercosis in Vellore district, India; Neurology. 2006: 67:2135-2139.

12. Prasad K N, Prasad A, Gupta R K, Pandey C M and Uttam S. Prevalence and associated risk factors of $T$. solium taeniasis in a rural pig farming community of North India; Trans. R. Soc. Trop. Med. Hyg. 2007:101:1241-1247

13. Life cycle image accessed from https://www.cdc.gov/dpdx/cysticercosis/index.html( on 2nd September,2017). 
14. Mamkin I et al., Taenia solium Neurocysticercosis. N Engl J Med 2007; 357:1666-1667

15. Rajshekhar V, Chandy MJ. Comparative study of $\mathrm{CT}$ and MRI in patients with seizures and a solitary cerebral cysticercus granuloma. Neuroradiology. 1996; 38: 542-6.

16. Govindappa SS, Narayanan JP, Krishnamoorthy VM, Shastry CH, Balasubramaniam A, Krishna SS. Improved detection of intraventricularcysticercal cysts with the use of three-dimensional constructive interference in steady state MR sequences. Am J Neuroradiol 2000; 21: 679-84.

17. Prasad K N, Prasad A, Gupta R K. Nath K, Pradhan $S$, Tripathi $M$ and Pandey C M Neurocysticercosis in Patients with Active Epilepsy From a Pig Farming Community; Trans. R. Soc. Trop. Med. Hyg. 2009; 103:144-150

18. H. H. Garcia, A. E. Gonzalez, R. H. Gilman et al., Short report: transient antibody response in Taenia solium infection in field conditions - a major contributor to high seroprevalence. Am. J. Trop. Med. Hyg. 2001; 65(1): 31-32.

19. R. J. Bobes, M. Hern'andez, C. M'arquez et al., "Subarachnoidal and intraventricular human neurocysticercosis: application of an antigen detection assay for the diagnosis and follow-up," Tropical Medicine and International Health, 2006: 11(6): 943-950.

20. S. Rodriguez, P. Dorny, V. C. W. Tsang et al., "Detection of Taenia solium antigens and anti-T. solium antibodies in paired serum and cerebrospinal fluid samples from patients with intraparenchymal or extraparenchymal neurocysticercosis," Journal of Infectious Diseases, 2009:199(9):1345-1352.

21. Tsang VCW, Brand JA, Boyer AE. An enzymelinked immunoelectrotransfer blot assay and glycoprotein antigens for diagnosing human cysticercosis (Taenia solium). J Infect Dis 1989; 159: 50-9.

22. Prabhakaran V, Raghava MV, Rajshekhar V, Muliyil J, Oommen A. Seroprevalence of Taenia solium antibodies in Vellore district, South India. Trans R Soc Trop Med Hyg., 2008; 102: 246-50.
23. A. Carpio, "Diagnostic criteria for human cysticercosis," Journal of the Neurological Sciences, 1998: 161(2): 185-188.

24. E. C. Bueno, A. J. Vaz, L. Machado, and J. A. Livramento. Neurocysticercosis: detection of $\mathrm{IgG}$, $\operatorname{IgA}$ and $\operatorname{IgE}$ antibodies in cerebrospinal fluid, serum and saliva samples by ELISA with Taenia solium and Taenia crassiceps antigens. Arquivos de NeuroPsiquiatria, 2000; 58(1):18-24.

25. Michelet L. et al., Human Neurocysticercosis: Comparison of Different Diagnostic Tests Using Cerebrospinal Fluid. Journal of Clinical Microbiology. 2011. Vol. 49(1): 195-200.

26. Biswas, R.; Parija, S.C. and Narayan, S.K. - DotELISA for the diagnosis of neurocysticercosis. Rev. Inst. Med. trop. S. Paulo. 2004; 46(5):249-252.

27. Coral-Almeida M, Gabriël S, Abatih EN, Praet N, Benitez W, Dorny P. Taenia solium Human Cysticercosis: A Systematic Review of Seroepidemiological Data from Endemic Zones around the World. Torgerson PR, ed. PLoS Neglected Tropical Diseases. 2015; 9(7):e0003919. doi:10.1371/journal.pntd.0003919.

28. Zamora H, Castillo Y, Garcia HH, Pretell J, Rodriguez S, Dorny P, et al., Drop in antigen levels following successful treatment of subarachnoid neurocysticercosis. Am J Trop Med Hyg 2005; 73: S41.

29. Serpa $\mathbf{J}$ et al., Advances in the diagnosis and management of neurocysticercosis. Expert Review Of Anti-Infective Therapy. $2006: 4(6)$.

30. Osvaldo Massaiti Takayanagui, Newton Satoru Odashima, Pierina S Bonato, Jose Eduardo Lima, and Vera Lucia Lanchote. Medical management of neurocysticercosis. Expert Opinion On Pharmacotherapy. 2011:12(18).

31. CoronaT.et al., Single-Day Praziquantel Therapy for Neurocysticercosis. N Engl J Med.1996; 334:125.

32. Del Brutto OH. Clinical management of neurocysticercosis. Expert Review of Neurotherapeutics 2014:14(4).

33. Garcia et al., Current consensus guidelines for treatment of neurocysticercosis. Clin. Microbiol. Rev. 2002: 747-756

\section{How to cite this article:}

Ramandeep Kaur, Harit Kumar, Shalini Pathania, and Nitin Goel Insan. 2017. Neurocysticercosis: Trends in Diagnosis and Management. Int.J.Curr.Res.Aca.Rev. 5(10), 8-14.

doi: https://doi.org/10.20546/ijcrar.2017.510.002 